\title{
Debatte
}

\section{Der Kulturkampf geht weiter}

Werner Abelshauser

Alle rufen nach neuen Regeln für die Wirtschaft. Der Schock der Finanzmarktkrise stellt die Imperien, deren Spielregeln gerade versagt haben, wie die USA oder China, vor große Herausforderungen. Die Europäer haben es da einfacher. Sie können auf bewährte Alternativen zurückgreifen auch wenn es lange her ist, dass die „rheinischen" Spielarten des Kapitalismus, wie die sprichwörtliche Deutschland $A G$ oder der capitalisme à la française unangefochten waren. Seit den 1990er Jahren schienen sie endgültig in die Defensive geraten und konnten sich nur noch mühsam gegen die vermeintliche Überlegenheit eines globalen Standardkapitalismus behaupten, der im Begriff war, das europäische Wirtschaftsmodell - wie andere varieties of $\mathrm{ca}$ pitalism auch - gleichzuschalten. Nach dem Ausbruch der Finanzmarktkrise könnten die Karten allerdings neu gemischt werden. Je mehr sein großer Widersacher in der Krise an Strahlkraft verliert und Verantwortung für die „Versumpfung des Kapitalismus" (Walter Eucken) übernehmen muss, desto mehr gewinnt das europäische Modell im Allgemeinen und das deutsche im Besonderen seine wirtschaftliche Reputation zurück. Immerhin hatten selbst kritische Beobachter, wie der französische Spitzenmanager Michel Albert, im capitalisme rhénane, der - von Skandinavien bis Norditalien, von der Seine bis an die Oder - zum gemeinsamen europäischen Erbe gehöre, nach dem Zusammenbruch des Ostblocks die weltweit erfolgreichste Variante der Marktwirtschaft gesehen. Albert fügte aber selbst skeptisch hinzu, dass der Rheinische Kapitalismus den politischen und medialen Kulturkampf mit seinem amerikanischen Widersacher wohl dennoch verlieren würde. Bis vor Kurzem sah es so aus, als würde er Recht behalten. Je rascher die Globalisierung voranschritt, desto stärker setzte sich der FinanzmarktKapitalismus in seiner Variante als share holder value capitalism durch, neben dem die „rheinischen“ Varianten, die dem Prin- zip des stakeholder value verpflichtet sind, immer mehr verblassten. Gleichzeitig brachte er neue wirtschaftliche Funktionseliten hervor, die als „Eigentümer ohne Risiko" (Paul Windolf) im Begriff waren, den Primat der Wirtschaft durchzusetzen.

\section{WORUM ES GEHT}

Erst die Finanzmarktkrise hat diese wirtschaftliche Usurpation des Gemeinwesens delegitimiert und den Blick für die Vorzüge des europäischen Modells wieder geschärft. Seine Varianten leiden nämlich keineswegs unter einem Mangel an komparativen Vorteilen, der sie obsolet werden ließe. Ganz im Gegenteil. Die deutsche Wirtschaft hat seit mehr als 100 Jahren die Herausforderung der Verwissenschaftlichung der Produktion und der Globalisierung angenommen und sich lukrative Märkte erobert, auf denen sie auch heute noch außerordentlich erfolgreich ist. Es sind dies vor allem die Märkte für diversifizierte Qualitätsprodukte. Darunter ist nachindustrielle Maßschneiderei zu verstehen, wie z. B. intelligente Maschinen mit individuellem Innenleben, Anlagenbau, anwendungstechnisch veredelte Produkte und Dienstleistungen aller Art, Verfahrenstechnik auf allen Gebieten oder auch hochwertige Fahrzeuge. Darin ist der deutsche Export kaum zu schlagen. Um in diesem großen und wichtigen Marktsegment wettbewerbsfähig zu sein, bedarf es eines institutionellen Rahmens, der auf diese Produktionsweise abgestimmt ist. Von wegen freie Standortwahl in der globalen Wirtschaft! Nur weil die europäische Wirtschaft über spezifische Institutionen verfügt und damit über komparative institutionelle Kostenvorteile im Finanzierungssystem, in der corporate governance, in den Arbeitsbeziehungen, in der Interessenpolitik, im Branchensystem und im Ausbildungswesen, beherrscht sie bis heute die Weltmärkte für diversifizierte Qualitätsproduktion. Allein die deutsche Exportwirtschaft liegt auf rund $40 \%$ der 750 im internationalen Handelsverzeichnis SITC (Standard international Trade Classification) ausgewiesenen Weltmärkte in der Spitze. Und weil sie auf diesen Märkten führend ist, braucht sie diesen institutionellen Rahmen samt seinen nicht immer einfachen Regeln.

Wenn dieser institutionelle Rahmen gleichwohl zur Disposition stand, ist dies nicht leicht zu verstehen. Nicht, dass die Bedeutung der diversifizierten Qualitätsproduktion auf den Märkten abgenommen hätte. Im Gegenteil, sie wächst. Nicht, dass ihre weltweite Wettbewerbsfähigkeit schwinden würde. Im Gegenteil, sie ist kaum noch zu steigern. Die Probleme liegen woanders. Das deutsche Produktionsregime scheint außerstande, die seit Jahren anhaltende Massenarbeitslosigkeit wirksam zu bekämpfen. Einer der wichtigsten Gründe für diese Unfähigkeit liegt auf der Hand: Fast ein Drittel der deutschen Erwerbstätigen verfügt über keine oder nur über einfache Qualifikationen und steht deshalb im globalen Verdrängungswettbewerb. Dieser für die deutschen Verhältnisse zu hohe Anteil Niedrigqualifizierter hat historische Gründe und ist uns als Erbe des fordistischen Zwischenspiels in der Wirtschaft der 1950er und 60er Jahre überkommen. ${ }^{1}$

\footnotetext{
1 Mehr dazu: W. Abelshauser (2009): Strukturelle Arbeitslosigkeit: Eine Diagnose aus historischer Perspektive, in: Jahrbuch für Wirtschaftsgeschichte $2009 / 2$, S. 221-234.
}

Werner Abelshauser, Prof. Dr. phil., Dipl.-
Volkswirt, lehrt Wirtschaftsgeschichte an der
Universität Bielefeld. Co-Direktor des dorti-
gen Instituts für Weltgesellschaft und des
Instituts für Wissenschafts- und Technikfor-
schung. Arbeitsschwerpunkte: Weltmarkt,
institutioneller Wandel, historische Unter-
nehmensforschung.
email: Werner.Abelshauser@uni-bielefeld.de


Die Kritik setzt aber auch tiefer an. Es fehlt der deutschen Wirtschaft nach verbreiteter Meinung zwar nicht an Innovationsfähigkeit im Allgemeinen, aber doch an Neuerungskraft auf bestimmten Märkten, von denen alle Welt annimmt, sie seien die zukunftsträchtigen. Auch wenn der deutsche Export insgesamt erfolgreich ist, muss diese Kritik sehr ernst genommen werden. Die deutsche Exportwirtschaft liegt nämlich auf dem Sektor für Informationstechnologie und auf anderen hochinnovativen Märkten nur im oberen Mittelfeld. An der Antwort auf die Frage, wie dieser Innovationsschwäche abgeholfen werden könnte, scheiden sich die Geister. Die einen setzen auf Reformen des bestehenden sozialen Systems der Produktion, um es von Schlacken zu befreien. Die anderen halten gerade dieses System für obsolet und daher einen Neustart für zwingend. Sie plädieren dafür, das bestehende gegen ein neues Produktionsregime mit anderen, vor allem aber weniger wirtschaftlichen Spielregeln auszutauschen, für die zumeist die USA als Vorbild dienen. Dies könnte auf schwachen Märkten vielleicht Abhilfe schaffen, ginge aber möglicherweise zulasten der Märkte für diversifizierte Qualitätsproduktion, von denen zwei Drittel der Erwerbstätigen (und der Unternehmen) sehr gut leben. Hier liegt der Kern des erbitterten Kulturkampfes, der jetzt in eine neue Phase tritt.

\section{WOZU KORPORATIVE MARKT- WIRTSCHAFT GUT IST}

Die meisten deutschen Unternehmen brauchen diese Wirtschaftskultur wie die Luft zum Atmen. Darunter ist die Art und Weise zu verstehen, wie die Wirtschaft in den genannten Funktionsbereichen organisiert und in die Gesamtgesellschaft eingebettet ist. Der Geist, der diesen organisatorischen Rahmen ausfüllt, lebt von den Institutionen, die als weithin akzeptierte Denk- und Handlungsweisen zu Spielregeln geronnen sind. Diese Institutionen standen einmal für die Kardinaltugenden deutscher Unternehmer (und ihrer wirtschaftlichen Mitspieler) und beanspruchen noch heute im überwiegenden Teil der deutschen Wirtschaft Geltung: Autonomiebewusstsein, Selbstverwaltung, Kooperationsbereitschaft und Soziabilität, also die Fähigkeit zur spontanen vertrauensvollen Zusammenarbeit, um sich im Wettbewerb auf wichtigen Märkten komparative
Vorteile zu sichern. Diese institutionelle und organisatorische Konstellation der Marktwirtschaft kennzeichnet eine Wirtschaft, in der weder das Individuum noch der Staat den Ton angibt. Korporative Marktwirtschaft - wie man sie nennen könnte - ist vielmehr in die dichte, historisch gewachsene Landschaft von Institutionen und Organisationen gebettet, deren Akteure in der Zivilgesellschaft (den Hegelschen Korporationen) zwischen diesen beiden Polen, Individuum und Staat, zu Hause sind.

Was diese Wirtschaft leisten kann und was nicht, ist weniger von Entscheidungen der Politik (oder auch der neuen wirtschaftlichen Eliten) abhängig als von den historisch gewachsenen Erfahrungen auf Märkten und mit Organisationen, die als Unternehmens- oder Wirtschaftskultur die Handlungsspielräume Erfolg versprechender Strategien in den Unternehmen und in der Wirtschaftspolitik abstecken. Aus der historischen Unternehmensforschung wissen wir, dass der Versuch, gegen die eigene Unternehmenskultur aus einem gewachsenen Portfolio auszubrechen, um auf schwächeren Märkten besser zu reüssieren, fehlschlagen kann und in der Vergangenheit oft genug gescheitert ist. Deshalb konzentrieren sich immer mehr Unternehmen auf ihr Kerngeschäft. Sie wollen ihre Stärken voll nutzen und von dieser sicheren Basis ausgehend behutsam, sozusagen auf Sicht, auch schwächere Märkte erobern. Was aus mikroökonomischer Perspektive richtig ist, muss für die Gesamtwirtschaft nicht falsch sein. Den Ländern des Rheinischen Kapitalismus mit ihrer mächtigen Wirtschaftskultur wäre deshalb zu empfehlen, die komparativen institutionellen Vorteile der eigenen Produktionsweise ordnungspolitisch abzusichern. Langfristig akkumulierte Institutionen lassen sich zwar rasch zerschlagen, neue Spielregeln und Organisationsformen der Wirtschaft, die komparative Kostenvorteile schaffen, aber nur langsam aufbauen. Der Erfolg einer solchen Radikaloperation müsste ungewiss bleiben.

Insbesondere für Unternehmen, die als global players am Weltmarkt operieren, bietet sich freilich die Alternative, allein auf Märkte und Hierarchien zu bauen, um flexibler zu agieren und den Gewinn kurzfristig zu maximieren. Die Entscheidung zum Ausstieg aus der korporativen Marktwirtschaft liegt dabei nicht in der Willkür der Unternehmen. Je mehr der internationale Kapitalmarkt von Regeln beherrscht wird, die eine langfristige unternehmerische Perspektive erschweren und tief in die Herrschafts- und Lenkungsverhältnisse der Unternehmen eingreifen, desto mehr stehen auch deutsche Unternehmen vor einem Dilemma. Sollen sie die neuen Spielregeln akzeptieren und darüber den Bruch mit der eigenen Unternehmenskultur riskieren, sodass sie ihre komparativen institutionellen Vorteile auf dem Weltmarkt verlieren? Oder sollen Unternehmen ihre Stärken weiter ausbauen und wo nötig wiederherstellen - auch wenn sie Gefahr laufen, auf manche Vorzüge, die ein funktionierender internationaler Kapitalmarkt bietet, verzichten zu müssen?

\section{WAS NICHT GEHT}

Es spricht in Europa einiges für eine an den Stärken der eigenen Unternehmenskultur ausgerichtete Ordnungspolitik. Denn wer auf Märkten für diversifizierte Qualitätsproduktion operiert, ist auf ,geduldiges Kapital“ ebenso angewiesen, wie auf engagierte, qualifizierte Mitarbeiter. Wer aber der bedingungslosen Anpassung an die Spielregeln des Finanzmarkt-Kapitalismus den Vorzug gibt, sollte wenigstens ihre Risiken kennen. Der gescheiterte Versuch der Deutsche Börse AG, die London Stock Exchange zu übernehmen, bietet dafür ein lehrreiches Beispiel. Ausgerechnet die Vorkämpfer für eine neue Aktienkultur in Deutschland, der frühere Börsenchef Werner Seifert und sein Aufsichtsratsvorsitzender, der ehemalige Sprecher der Deutschen Bank Rolf-Ernst Breuer, mussten erkennen, wie eng der unternehmerische Handlungsspielraum werden kann, wenn ,unfreundliche" Aktionäre dem Vorstand ins Handwerk pfuschen. Noch während der Übernahme-Operation gewannen neue Aktionärsgruppen in der Hauptversammlung die Mehrheit, die das anvisierte mittelfristige Wachstum des Gewinns je Aktie niedriger bewerteten als eine cash-Ausschüttung hier und heute. Die neuen Großaktionäre Atticus Capital LP (New York) und The Children's Investment Fund (TCI, London), klassische Hedge-Fonds mit typischer „Heuschrecken“-Strategie, hielten ein knappes Fünftel der Aktien. Weil die deutschen Banken zuvor ihre Kontrollmehrheit aufgegeben hatten, reichte dies aus, um zusammen mit anderen Anhängern des Shareholder-Value-Prinzips unter den Aktionären im Frühjahr 2005 den Sturz von Seifert und Breuer herbeizuführen und 
so den Kauf der Londoner Börse zu verhindern. Mittelabflüsse, die nicht in die Taschen der Aktionäre flossen, wurden seitdem rigoros unterbunden. Unter diesen Bedingungen unternehmerisch langfristig $\mathrm{zu}$ handeln, wie es der Produktionsweise des Rheinischen Kapitalismus angemessen ist, schien nahezu unmöglich. Erst unter dem Eindruck der 2007 einsetzenden Finanzmarktkrise mussten sich die HedgeFonds zurückziehen, bevor es ihnen vollends gelungen war, das diversifizierte $\mathrm{Ge}$ schäftsmodell der Deutsche Börse zu zerschlagen und das Unternehmen in seinen Einzelteilen meistbietend zu verkaufen. Die mehrheitliche Kontrolle ausländischer Fonds-Gesellschaften über das Aktienkapital der 30 DAX-Unternehmen, die unabhängig von diesem Beispiel noch immer gegeben ist, hat einen tieferen Grund. Während in Deutschland das Sozialvermögen der privaten Rentenanwartschaften von rund 7 Bio. $€(2005)$ am Kapitalmarkt vorbei seinen Empfänger findet, müssen in den USA rund 35 Bio. $€$ über private Fonds-Gesellschaften an den internationalen Kapitalmärkten Anlage suchen. Die Kapitalmarktregeln der europäischen Sozialstaaten werden somit paradoxerweise von den Anlegern bestimmt, die aus MarktStaaten (Philip Bobbitt) kommen und unter Konkurrenzdruck extreme Formen des short termism praktizieren. Für die europäische Produktionsweise der nachindustriellen Maßschneiderei ist dies Gift. Sie braucht eine langfristige unternehmerische Perspektive.

\section{WER BÄNDIGT DAS MONSTER?}

Wenn sich das Modell der korporativen Marktwirtschaft auch unter starkem Druck bisher als widerstandsfähig erwiesen hat, liegt dies gewiß nicht in erster Linie an der Einsicht der Dax-Vorstände in die Überlegenheit europäischer Spielregeln über die Dogmen des Standardkapitalismus. Ihre wahren Verfechter sind vielmehr die kleinen und mittleren Unternehmen, die den Löwenanteil zur Beschäftigung und zum Volkseinkommen in Deutschland und Eu- ropa beitragen. Sie sind auf diese Spielregeln nicht nur angewiesen, um im globalen Wettbewerb zu bestehen; sie machen auch unmittelbare und meist positive Erfahrungen mit ihrer Anwendung. Sie geraten deshalb nicht so leicht in Versuchung, auf modische Konzepte zu setzen, deren Horizont oft nicht weit über die kurzfristigen Ziele angestellter Manager in Großunternehmen oder institutioneller Anleger auf dem Kapitalmarkt hinausreicht. Dies gilt nicht nur, aber vor allem für die - meist selbstständigen - Unternehmer, die für die Funktionsfähigkeit des knappen Dutzends regionaler Verbundstandorte („Cluster“) verantwortlich sind, die das Rückgrat der deutschen Außenwirtschaft bilden. Dass sich aber auch kleine und mittlere Unternehmen nicht immer ihrer eigenen Interessen bewusst sind, zeigt ihre sinkende Neigung, das duale System der Berufsbildung zu nutzen. Es ist fast ein Gemeinplatz: Arbeitgeber, die zu wenig in das menschliche Vermögen investieren und Deutschland zum Niedriglohnland machen wollen, schaden sich selbst und der ganzen Wirtschaft. Ausbildung, die den Bedürfnissen diversifizierter Qualitätsproduktion entspricht, ist eine viel gerühmte deutsche Spezialität. Wer mit Blick auf die amerikanische Praxis glaubt, er müsse nicht ausbilden und könne sich kostengünstiger „am Markt“ mit Facharbeitern und Technikern eindecken, macht einen Denkfehler. Qualifizierte Arbeitskräfte, die zur nachindustriellen Maßschneiderei taugen, sind auf dem Weltmarkt nicht zu haben. Ihre Ausbildung gehört zu den komparativen institutionellen Kostenvorteilen der deutschen Wirtschaft. Wenn Unternehmen zu wenig Gebrauch davon machen, muss Ordnungspolitik nachhelfen. Die Krise könnte auch hier zu neuen Einsichten führen.

Unternehmen werden nur dann ihrer Verantwortung gerecht werden können, wenn sie nicht nur wissen, wie sie sich verhalten müssen, damit das System funktioniert und dies auch wollen, sondern wenn alle Voraussetzungen erfüllt sind, damit sie es auch können. Dies bringt die Politik ins
Spiel, um daraus konkrete Forderungen an die Sozialpflichtigkeit des Eigentums abzuleiten. In diesem Sinne gehört es zu den legitimen ordnungspolitischen Aufgaben des Staates und zu den Vorrechten einer demokratisch verfassten Öffentlichkeit, auch die Unternehmen auf ihre Verantwortung im sozialen System der Produktion zu verpflichten. Dies setzt ordnungspolitische Grundsatzentscheidungen voraus - zumal auch keine der alternativen Strategien auf die Ressource Politik verzichten kann. Der Staat ist auch gefragt, wenn es um die Bändigung des „Monsters“ (Horst Köhler) geht, zu dem sich die internationalen Finanzmärkte seit den 1970er Jahren entwickelt haben. So bezieht kein Geringerer als der deutsche Bundespräsident, der zuvor die wichtige Position des Direktors des Internationalen Währungsfonds innehatte, eindeutig Stellung im Kampf der Wirtschaftskulturen. Er fordert die Deutschen auf, ihren Finanzsektor „strategisch“ zu überprüfen und empfiehlt der Politik „ein selbstbewusstes Eintreten für die kontinentaleuropäische Finanz- und Bankkultur“. Die Kritik an der aus der Kontrolle geratenen Verfassung des globalen Kapitalmarktes hat schon Tradition. Auf der Weltwirtschaftskonferenz in Venedig eröffnete $\mathrm{Hel}$ mut Schmidt 1980 in seiner Rolle als „Weltökonom“ den Reigen der Kritiker, als er die rudimentäre Weltwirtschaftsregierung der G 7 dazu aufforderte, endlich die Herrschaft über die Kapitalmärkte zurückzugewinnen. Die Aktualität seiner Warnung weist auf eine Irridenta der internationalen Finanzpolitik hin, deren Lösung über Glanz und Elend der Weltwirtschaft entscheiden kann. Hinter der Schwierigkeit, den neuen Leviathan zu bändigen, der an die Stelle des Staates als ,sterblicher Gott" (Thomas Hobbes) getreten ist, verbirgt sich aber der eigentliche Kern des Problems: internationale wirtschaftliche Interessenpolitik. Sie fordert den Kulturkampf heraus, in dem sich die Interessen aus divergenten sozialen Produktionssystemen auf dem Weltmarkt im Streit um die Spielregeln gegenüberstehen. 